\title{
The gut microbiota of nonalcoholic fatty liver disease: current methods and their interpretation
}

\author{
Niels van Best ${ }^{1} \cdot$ Peter L. Jansen ${ }^{1} \cdot$ Sander S. Rensen ${ }^{1}$
}

Received: 12 January 2015/ Accepted: 19 May 2015/Published online: 12 June 2015

(c) The Author(s) 2015. This article is published with open access at Springerlink.com

\begin{abstract}
The role of intestinal bacteria in the pathogenesis of nonalcoholic fatty liver disease is increasingly acknowledged. Recently developed microbial profiling techniques are beginning to shed light on the nature of gut microbiota alterations in nonalcoholic fatty liver disease. In this review, we summarize the gut microbiota composition changes that have been reported during different stages of human nonalcoholic fatty liver disease, and highlight the relation between bile acids and gut bacteria in this context. In addition, we discuss the different methodologies used in microbiota analyses as well as the interpretation of microbiota data. Whereas the currently available studies have provided useful information, future large-scale prospective studies with carefully phenotyped subjects and sequential sampling will be required to demonstrate a causal role of gut microbiota changes in the etiology of nonalcoholic fatty liver disease.
\end{abstract}

Keywords Gut microbiota $\cdot$ Steatosis - Steatohepatitis · Fibrosis · Cirrhosis · Bile acids

\section{Introduction}

Nonalcoholic fatty liver disease (NAFLD), the hepatic manifestation of the metabolic syndrome [1], is characterized by hepatic fat accumulation in the absence of significant alcohol consumption, viral infection, or other liver

Sander S. Rensen

s.rensen@maastrichtuniversity.nl

1 Department of Surgery, NUTRIM School of Nutrition and Translational Research in Metabolism, Maastricht University Medical Center, Maastricht, The Netherlands disorders [2]. NAFLD ranges from simple steatosis to inflammatory nonalcoholic steatohepatitis (NASH), with or without fibrosis. It is the most common liver disorder worldwide, and has an increasing prevalence. NASH, but not simple steatosis, frequently progresses to life threatening disorders such as cirrhosis and hepatocellular carcinoma (HCC) [3].

NAFLD pathophysiology is multifactorial, involving ecological, genetic, and metabolic factors such as limited physical activity, high energy intake, and a dysbalanced diet (e.g. too much fructose and/or saturated fat) [4]. Together with epigenetic factors, this promotes insulin resistance and hepatic fat accumulation [2, 5]. Progression towards inflammation of the steatotic liver was initially proposed to be related to endotoxemia as a result of increased gut permeability by Brun et al. [6] and Wigg et al. [7]. Subsequently, evidence accumulated that intestinal microbiota plays an important part in the pathogenesis of NAFLD [8-10]. Microbial profiling techniques developed in the past few years enabled major advances in our understanding of alterations of the gut microbiota and the role of gut bacteria in the development of NAFLD [11].

This review summarizes these recent findings, focusing on gut microbiota composition changes during the different stages of human NAFLD, and paying particular attention to the methodologies used in microbiota analyses as well as their interpretation.

\section{Microbiota composition in NAFLD}

\section{Steatosis and steatohepatitis}

There are only a limited number of studies that have examined microbiota composition in patients with simple 
steatosis or NASH, and these have very dissimilar results (Table 1). First of all, patients with NASH were recently shown to have a decreased abundance of bacteria belonging to the phylum Bacteroidetes compared to subjects with simple steatosis and healthy individuals as shown by qPCR [9]. In contrast, studies using sequencing techniques showed an increase of Bacteroides, one of the most important genera within the Bacteroidetes phylum, and a decrease of Firmicutes in NASH patients as compared to healthy subjects [8]. The lower representation of Firmicutes in NASH patients was especially due to a reduced abundance of the Lachnospiraceae and Ruminococcaceae families. However, another study demonstrated an increase of Lachnospiraceae and Lactobacillaceae in NAFLD patients, albeit without distinguishing

Table 1 Significant microbiota composition changes in nonalcoholic liver disease

\begin{tabular}{|c|c|c|c|c|}
\hline Disease comparison & Samples & $\begin{array}{l}\text { Microbiota variations } \\
\text { (family_genus) }\end{array}$ & Techniques & References \\
\hline $\begin{array}{l}\text { Non-NASH cirrhotic patients }(n=181) \text { versus NASH } \\
\text { cirrhotic patients }(n=32)\end{array}$ & Stool & $\begin{array}{l}\uparrow \text { Bacterioidaceae } \\
\uparrow \text { Porphyromonadaceae } \\
\downarrow \text { Veillonellaceae }\end{array}$ & $\begin{array}{l}\text { 16S rRNA MT } \\
\text { pyrosequencing }\end{array}$ & {$[13]$} \\
\hline Healthy $(n=17)$ versus NASH $(n=22)$ & Stool & $\downarrow$ Phylum: Bacteroidetes & qPCR & [7] \\
\hline Simple steatosis $(n=11)$ versus NASH $(n=22)$ & Stool & $\begin{array}{l}\downarrow \text { Phylum: Bacteroidetes } \\
\uparrow C \text {. coccoides }\end{array}$ & qPCR & [7] \\
\hline Healthy $(n=30)$ versus obese NAFLD $(n=30)$ & Stool & $\begin{array}{l}\uparrow \text { Veillonellaceae } \\
\uparrow \text { Kiloniellaceae } \\
\uparrow \text { Pasteurellaceae } \\
\uparrow \text { Lactobacillaceae } \\
\uparrow \text { Lachnospiraceae } \\
\downarrow \text { Ruminococcaceae } \\
\downarrow \text { Porphyromonadaceae } \\
\uparrow \text { Lactobacillaceae_Lactobacillus } \\
\uparrow \text { Lachnospiraceae_Dorea } \\
\uparrow \text { Lachnospiraceae_Robinsoniella } \\
\uparrow \text { Lachnospiraceae_Roseburia } \\
\downarrow \text { Ruminococcaceae_Oscillibacter }\end{array}$ & $\begin{array}{l}\text { 16S rRNA MT } \\
\text { pyrosequencing }\end{array}$ & {$[8]$} \\
\hline Obese $(n=25)$ versus NASH $(n=22)$ children & Stool & $\begin{array}{l}\uparrow \text { Enterobacteriaceae } \\
\uparrow E n t e r o b a c t e r i a c e a e \_E s c h e r i c h i a\end{array}$ & $\begin{array}{l}\text { 16S rRNA MT } \\
\text { pyrosequencing }\end{array}$ & {$[6]$} \\
\hline Healthy $(n=16)$ versus NASH $(n=22)$ children & Stool & $\begin{array}{l}\uparrow \text { Enterobacteriaceae } \\
\uparrow \text { Enterobacteriaceae_Escherichia } \\
\downarrow \text { Bifidobacteriaceae_Bifidobacterium } \\
\downarrow \text { Bifidobacteriaceae } \\
\uparrow \text { Prevotellaceae } \\
\uparrow \text { Prevotellaceae_Prevotella } \\
\downarrow \text { Rikenellaceae } \\
\downarrow \text { Rikenellaceae_Alistipes } \\
\uparrow \text { Clostridiales XI_Peptoniphilus } \\
\downarrow \text { Lachnospiraceae } \\
\downarrow \text { Lachnospiraceae_Blautia } \\
\downarrow \text { Lachnospiraceae_Coprococcus } \\
\downarrow \text { Eubacteriaceae_Eubacterium } \\
\downarrow \text { Lachnospiraceae_Roseburia } \\
\downarrow \text { Ruminococcaceae } \\
\downarrow \text { Ruminococcaceae_Oscillospira } \\
\downarrow \text { Ruminococcaceae_Ruminococcus } \\
\downarrow \text { Ruminococcaceae_Unclassified } \\
\uparrow \text { Alcaligenaceae }\end{array}$ & $\begin{array}{l}\text { 16S rRNA MT } \\
\text { pyrosequencing }\end{array}$ & {$[6]$} \\
\hline
\end{tabular}


between simple steatosis and NASH [10]. Both studies observed a decrease in members of the Ruminococcaceae family in NASH $[8,10]$. The apparent lack of consistent changes of gut microbiota composition in NASH is further exemplified by two recent studies, showing either an overrepresentation of the genus Escherichia from the Enterobacteriaceae family in subjects with NASH [8] or no difference in Escherichia coli abundance in NASH patients compared to subjects with simple steatosis [9]. Despite the lack of consistent NAFLD-related gut microbiota changes, the possible overgrowth of these ethanol-producing bacteria may underlie the increased circulating ethanol levels in NASH [8]. The endogenous production of ethanol might, in turn, contribute to the formation of free fatty acids and oxidative stress (Fig. 1), further underscoring the potential role of ethanol-producing bacteria in the pathogenesis of NAFLD.

Large scale trials, designed to identify alterations of microbiota composition in patients with simple steatosis versus NASH, are required to shed more light on the nature of the gut microbiota shifts characteristic of specific stages of NAFLD. These trials should also pay attention to the fact that most NAFLD patients are obese, since obesity itself is linked to gut microbiota composition changes, as reviewed elsewhere $[12,13]$. Future studies should ideally include obese non-NAFLD patients or non-obese NAFLD patients [14] to exclude the impact of obesity, or control for obesity in statistical analyses. It should be further noticed that many studies so far excluded all taxa with an abundance below $1 \%$. However, even low-abundant bacteria such as Akkermansia muciniphila have the potential to profoundly affect host metabolism [15]. In addition, without any fundamental direct evidence provided by fecal transplantation or antibiotic studies, one cannot exclude that the described alterations in the intestinal microbiota are a consequence rather than a cause of liver disease.

\section{Fibrosis and cirrhosis}

Surprisingly little evidence exists to date for an effect of gut microbiota on liver fibrosis. However, very recently, an elegant study was published showing that in a bile duct ligation-induced liver fibrosis model, transplantation of the

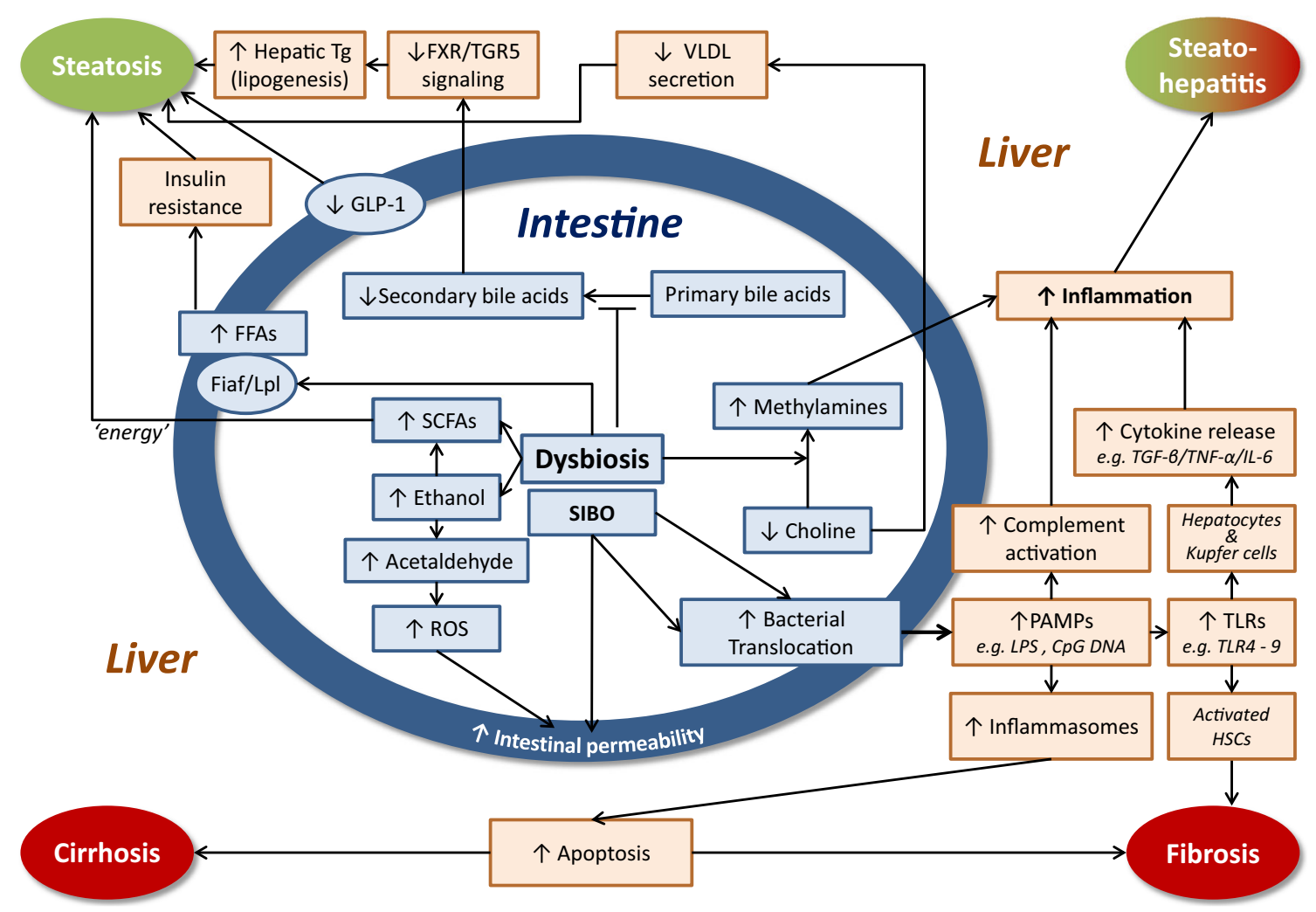

Fig. 1 Mechanisms by which gut bacteria affect the hallmarks of nonalcoholic fatty liver disease. SCFAs short-chain fatty acids, $P A M P s$ pathogen associated molecular patterns, $R O S$ reactive oxygen species, FFAs free fatty acids, $T g$ triglyceride, LPS lipopolysaccharide, TLR Toll-like receptor, SIBO small intestinal bacterial overgrowth, TGF- $\beta$ transforming growth factor- $\beta, I L-6$ interleukin6, TNF- $\alpha$ tumor necrosis factor- $\alpha$, HSCs hepatic stellate cells, Fiaf fasting induced adipocyte factor, $L p l$ lipoprotein lipase, $V L D L$ very low density lipoprotein, $G L P-1$ glucagon-like peptide-1 
gut microbiota of mice fed a high fat diet (HFD) aggravated fibrosis relative to transplanting gut microbiota of mice fed chow [16]. This was mainly attributable to an increased abundance of Gram-negative Proteobacteria; a marked decrease of Bifidobacteriaceae was also observed. Specific bacteria of both the Proteobacteria and Firmicutes phyla produce enzymes catalyzing choline conversion into methylamines [17]. The latter may promote liver inflammation via the portal vein, and decreased choline levels have already been associated with fibrosis progression [18]. However, future studies should ascertain to what extent the described microbiota composition changes in fibrosis affect choline metabolism.

Cirrhosis patients often have a higher proportion of potentially pathogenic bacteria and a reduction of autochthonous (resident) bacteria compared to healthy individuals (Table 2) [19-21]. Common differences include a decrease in families with the potential to convert primary into secondary bile salts (Lachnospiraceae and $R u$ minococcaceae) in cirrhosis, and overgrowth of the Gramnegative Enterobacteriaceae family, similar to what is observed in NASH [22]. This suggests that bile salts and endotoxin [23] may play a role in the pathogenesis of cirrhosis. Additional gut microbiota changes in patients with cirrhosis include a decrease in Clostridiales XIV and an increase in Enterococcaceae and Staphylococcaeae [19] as well as overgrowth of Veillonellaceae [21].

Another cohort of cirrhotic patients revealed a decrease of the genera Bacteroides, Eubacterium, and Alistipes, whereas Clostridium and Prevotella were increased compared to healthy controls [24]. However, the most abundantly enriched species in these cirrhotic patients belonged to the Streptococcus and Veillonella genera. Remarkably, these genera comprise oral species that might invade the gut and contribute to small-intestinal bacterial overgrowth, which frequently occurs in NASH and cirrhosis [7, 25]. Furthermore, the lower microbial richness and reduced abundance of butyrate-producing species with anti-inflammatory properties (F. prausnitzii, Coprococcus comes, Lachnospiraceae spp., Ruminoccaceae spp.) suggest that patients with cirrhosis have a less "healthy" microbiota $[24,26,27]$.

Cirrhotic patients with NASH further demonstrated a decrease in Veillellaceae and an increase in Bacteroidaceae and Porphyromonadaceae families compared to cirrhotic patients without NASH [19]. Although the abundance of Enterobacteriaceae was increased in NASH patients compared to healthy individuals, Enterobacteriaceae in NASH patients within a cirrhotic cohort were not affected. The latter might be due to the high proportion of Enterobacteriaceae in cirrhotic patients [19, 21, 22]. Interestingly, Bajaj and colleagues showed that the microbiome in cirrhotic patients with stable disease remains unaltered over time, suggesting that the composition of the microbiome can be used as a potential disease marker [19]. All in all, it appears that different stages of NAFLD are associated with different microbiota compositions, although these need to be better defined.

\section{Gut microbiota, bile acids, and hallmarks of NAFLD}

The mechanisms by which gut bacteria affect the various manifestations of NAFLD have been reviewed recently by Schnabl et al. [28]. The most relevant insights obtained in this burgeoning field of research are summarized in Fig. 1. In view of the accumulating evidence for the role of bile acids in the treatment of NAFLD [29], we will here focus on the intimate and reciprocal relation between bile acids and the gut microbiota.

Bacteria are needed for deconjugation, $7 \alpha$-dehydroxylation, and dehydrogenation of primary bile acids. Furthermore, the conversion of primary to secondary bile acids entirely depends on bacteria. Interestingly, germ-free mice have an increased bile acid synthesis in parallel with a decreased fecal bile acid output and an expanded circulating bile acid pool [30]. Thus, there appears to be a relation between the gut microbiota, bile acid synthesis in the liver, and bile acid uptake in the terminal ileum. Fibroblast growth factor 19 (FGF19; Fgf15 in rodents) plays a major role in this by linking events in the gut to metabolism in the liver [31, 32]. Upon activation of the Farnesoid X receptor (FXR) by bile acids, FGF19 is produced in the ileum and secreted into the portal circulation. In the liver, FGF19 action ultimately results in reduced transcription of Cyp7A1, the rate-limiting enzyme for bile acid synthesis [31].

Recent experiments illustrate the interdependence of gut microbiota and hepatic bile acid synthesis. Bile acids chenodeoxycholate (CDCA) and cholate (CA) act as FXR agonists, while tauro- $\alpha$-muricholic acid (TaMCA) and tauro- $\beta$-muricholic acid (T $\beta$ MCA) antagonize FXR [30, 33]. In germ-free mice, T $\beta M C A$ is produced relatively in excess over CA, suppressing the generation of Fgf15 thereby increasing primary bile acid synthesis [30], with T $\beta M C A$ in excess over CA. In conventional mice, the T $\beta M A / C A$ ratio is more in favor of $\mathrm{CA}$, limiting bile acid synthesis. This has implications for the actions of antibiotics. The administration of ampicillin to mice decreases Fgf15, thereby increasing Cyp7a1 expression and the synthesis of primary bile acids [30, 34]. Miyata et al. [35] explain the reduced Fgf15 expression by a lack of secondary bile acids in antibiotic-treated mice. A more likely explanation is that the T $\beta \mathrm{MCA} / \mathrm{CA}$ ratio increases under antibiotics, causing decreased Fgf15 expression and 
Table 2 Significant microbiota composition changes in cirrhosis

\begin{tabular}{|c|c|c|c|c|}
\hline Disease comparison & Samples & Microbiota variations (family_genus) & Techniques & References \\
\hline $\begin{array}{l}\text { Healthy }(n=25) \text { versus cirrhotic compensated outpatients } \\
\quad(n=121) \text {, cirrhotic decompensated outpatients } \\
(n=54) \text {, cirrhotic inpatients }(n=44)\end{array}$ & Stool & $\begin{array}{c}\downarrow \text { Clostridiales XIV } \\
\downarrow \text { Ruminococcaceae } \\
\quad \downarrow \text { Lachnospiraceae } \\
\uparrow \text { Enterococcaeae } \\
\uparrow \text { Staphylococcaceae } \\
\uparrow \text { Enterobacteriaceae }\end{array}$ & $\begin{array}{l}\text { 16S rRNA MT } \\
\text { Pyrosequencing }\end{array}$ & [13] \\
\hline Healthy $(n=17)$ versus liver cirrhosis $(n=36)$ & $\begin{array}{l}\text { Sigmoid } \\
\text { mucosa }\end{array}$ & $\begin{array}{l}\uparrow \text { Burkholderiaceae_Burkholderia } \\
\uparrow \text { Burkholderiaceae_Ralstonia } \\
\uparrow \text { Clostridiaceae_Clostridium } \\
\uparrow \text { Clostridiaceae_other } \\
\uparrow \text { Enterobacteriaceae_Proteus } \\
\uparrow \text { Enterococcaceae_Enterococcus } \\
\downarrow \text { Incertae Sedis XIV_other } \\
\downarrow \text { Lachnospiraceae_Dorea } \\
\downarrow \text { Lachnospiraceae_unclassified } \\
\downarrow \text { Ruminococcaceae_Subdoligranulum } \\
\downarrow \text { Veillonellaceae_Acidaminococcus }\end{array}$ & $\begin{array}{l}\text { 16S rRNA MT } \\
\text { Pyrosequencing }\end{array}$ & [14] \\
\hline Healthy $(n=14)$ versus liver cirrhosis $(n=47)$ & Stool & $\begin{array}{l}\uparrow \text { Enterobacteriaceae } \\
\uparrow \text { Veillonellaceae } \\
\downarrow \text { Lachonospiraceae } \\
\downarrow \text { Ruminococcaceae } \\
\downarrow \text { Ruminococcaceae_Blautia }\end{array}$ & $\begin{array}{l}\text { 16S rRNA MT } \\
\text { Pyrosequencing }\end{array}$ & {$[15]$} \\
\hline Healthy $(n=4)$ versus liver cirrhosis $(n=6)$ & & $\begin{array}{l}\uparrow \text { Enterobacteriaceae } \\
\uparrow E n t e r o c o c c u s\end{array}$ & RT-PCR & [16] \\
\hline Healthy $(n=98)$ versus liver cirrhosis $(n=83)$ & Stool & $\begin{array}{l}\downarrow \text { Bacteroidaceae_Bacteroides } \\
\downarrow \text { Eubacteriaceae_Eubacterium } \\
\downarrow \text { Rikenellaceae_Alistipes } \\
\uparrow \text { Veillonellaceae_Veillonella } \\
\uparrow \text { Streptococcaceae_Streptococcus } \\
\uparrow \text { Clostridiaceae_Clostridium } \\
\uparrow \text { Prevotellaceae_Prevotella }\end{array}$ & Illumina & [18] \\
\hline
\end{tabular}

increased bile acid synthesis [30]. Of note, humans do not produce T $\alpha \mathrm{MCA}$ and T $\beta \mathrm{MCA}$ and therefore the effect of antibiotics on bile acid synthesis in humans may not be the same as in mice.

Bile acids have a direct antimicrobial effect and affect microbiota composition [36, 37]. For example, administration of CA to rats induced outgrowth of bacteria in, for example, the genus of Clostridia. These efficiently transform primary bile acids into deoxycholic acid (DCA) by $7 \alpha$-dehydroxylation [37]. These changes are similar to the changes seen with a high fat diet. Furthermore, bile duct ligation in mice induces bacterial overgrowth, mucosal injury, and bacterial translocation [38, 39]. Lack of FXRmediated production of bacteriostatic angogenin 1 may play a role in this, but the details need to be elucidated. Patients with advanced cirrhosis have a reduced fecal concentration of total bile acids and a predominance of primary bile acids [21]. Advanced cirrhotics also have a higher abundance of Enterobacteriaceae and a decrease of Clostridia [19]. These intestinal bile acid alterations may contribute to changes of the microbiota. One can argue that in cirrhotics with a contracted bile acid pool, FXR activation in the ileum will be reduced. This leads to upregulation of the apical sodium-dependent bile salt transporter in the terminal ileum, reducing spill-over of bile acids from ileum to cecum. Bile acids in the cecum have a strong effect on $7 \alpha-$ dehydroxylating Clostridia [21]. Reduction of the cecal/colonic bile acid concentration therefore decreases conversion of primary bile acids into DCA and LCA (lithocholic acid).

In contrast, NAFLD-inducing high fat diets (HFD) in mice increase the conversion of primary to secondary bile 
acids. DCA has pro-inflammatory and DNA-damaging properties. Yoshimoto et al. [40] report that DCA induces a 'senescence-associated secretory phenotype' in hepatic stellate cells. Among the secretory products are IL-6 and PAI-1, factors known to induce cancer and obesity [41]. However, diet-induced obesity by itself is not enough to induce hepatocellular cancer (HCC) in mice; it was the combination of HFD and treatment with a carcinogen that was required. The role of DCA in this model was shown by administration of an inhibitor of $7 \alpha$-dehydroxylation, which decreased serum DCA concentration and HCC development. In contrast, adding DCA to the HFD increased HCC development [40], the ultimate consequence of NAFLD.

From these studies it is clear that bile acids are major players in the interaction between the gut microbiota and the host. Bile acids affect signaling paths, not only those mediated by FXR and FGF19, but also pathways regulated by the xenobiotic receptor PXR, the vitamin $\mathrm{D}$ receptor VDR, the G protein-coupled transmembrane receptor TGR, the muscarinic receptor, and the conjugated bile acid receptor [42]. These receptors affect metabolism in a wide variety of cells and organs both within and outside the enterohepatic circulation. For drug development, this new knowledge provides opportunities and challenges. Little attention has been paid yet to the effect of new potent FXR agonists like obeticholic acid on the microbiota. In view of the effects these drugs have on bile acid metabolism and FGF19 expression, they likely will also affect the microbiota. Furthermore, bile acid-mediated activation of TGR5 induces secretion of the glucose homeostasis regulating hormone glucagon-like peptide-1 (GLP-1) [43], which levels are decreased in NAFLD patients [44]. Other microbial metabolites such as indole and butyrate are also able to promote GLP-1 secretion [45, 46], and modulation of the gut microbiota by prebiotics or antibiotics affects GLP-1 secretion as well [46-48]. This further underscores the close interaction between the gut microbiota, bile acids, and gut hormones involved in metabolism.

\section{How to study and interpret microbiota}

To interpret the relevance of published data on microbiota in NAFLD, both the characterization of the disease and the type of microbial assays employed need to be taken into account. Importantly, the intestinal microbiota composition can be affected by even small changes in experimental methods at several steps from sample collection to statistical analysis, resulting in a different outcome of apparently identical studies. The most crucial technical and analytical aspects of microbial profiling, i.e. factors that may influence the results and/or result in possible bias, will be discussed here.

\section{Sampling and storage}

It is essential that sampling and storage methods do not modify microbiota composition by themselves. An important consideration in this respect concerns the type of samples collected, which are commonly stool or endoscopic biopsies. Stool samples are non-invasive and easy to obtain. However, the colonic mucosal microbiota has been shown to deviate considerably from stool microbiota, also in cirrhotic patients [49-51]. Moreover, whereas microbiota composition along the colon was considered homogenous only a decade ago [50, 51], recent highthroughput microbial profiling techniques revealed that the mucosal microbiota varies along the length of the gut [52, 53].

Immediate freezing of microbiota-containing samples is regarded the gold standard for long-term storage. However, this is a logistic challenge in large cohort studies which usually rely on fecal swabs [54]. Fecal swabs are commonly stored for short-term at room temperature in specific media in which obligate anaerobes can survive [55]. Recently, the impact of different sampling (fecal aliquots and fecal swabs) and storage techniques $\left(-80,-20,+4{ }^{\circ} \mathrm{C}\right.$ for 1 week and RT for $24 \mathrm{~h}$ ) on fecal microbiota composition was examined in healthy and diseased individuals [56]. Although no significant effect of storage temperature on microbiota composition was observed during transport (24 h), fecal swabs stored in Cary-Blair medium showed an enrichment of Ruminococcus and Enterobacteriaceae in comparison to fecal aliquots stored at $-80{ }^{\circ} \mathrm{C}$. Therefore, it is recommended to use a single uniform method within one study to minimize possible bias. Additionally, it is crucial to only compare results that have been obtained with the same sample methodology and from the same type of sample.

As for the design of studies, large-scale well-defined prospective cohorts of patients that have been carefully phenotyped are essential. In these studies, factors known to influence gut microbiota composition should be taken into account. In particular, diet and use of antibiotics, probiotics, and prebiotics should be well-documented. Furthermore, sex-specific differences in the human colonic microbiota have recently been shown [52] and should be considered when setting up microbiota studies.

\section{Microbial screening techniques}

The human gut mainly harbors strictly anaerobic microbial species that are difficult to culture. Therefore, several culture-independent techniques have been introduced in recent decades to analyze composition and complexity of the intestinal microbiota. These techniques include (quantitative) polymerase chain reaction [(q)PCR], PCR 
followed by denaturing gradient gel electrophoresis, fluorescent in situ hybridization (FISH), and DNA microarrays that hybridize ribosomal RNA (rRNA) sequences with probes.

Although these methods are useful for rapid microbial profiling, they usually do not provide detailed taxonomic data. For that reason, the most important techniques nowadays are high-throughput next-generation sequencing followed by bioinformatics analysis. These sequence methods are commonly based on analysis of the 16S rRNA gene which is present in all bacteria and archaea, consisting of nine unique hypervariable regions (V1-V9) and bordered by highly conserved regions [57]. Despite the fact that other genes have been suggested, sequencing of $16 \mathrm{~S}$ rRNA remains the gold standard to analyze the microbiota in view of the completeness of reference databases, low costs, and advanced bioinformatics software available. Different next-generation sequencers can be used to analyze the 16S rRNA gene. However, the majority utilizes either Illumina sequencing (Illumina Inc., San Diego, CA) or 454 pyrosequencing (Roche, Brandfort, CT). These widely popular approaches have distinct coverage ratios, sequence lengths, and construction mechanisms. 454 pyrosequencing generates longer sequences which increases taxonomic accuracy, whereas Illumina provides higher coverage at lower cost per sample [58]. It should be noted that the selection of primers, the number of sequences, costs, and aim of the experiment are strongly interdependent. Therefore, it is essential to balance these factors to achieve the optimum amount of information.

Although 16S sequencing is the most commonly used technology in microbiome studies, it is important to understand its limitations. Differences in DNA extraction methods, PCR errors, and discrepancy in 16S gene copy numbers all affect the proportions of bacteria detected [5961]. The latter leads to bias in the detection of unknown or unclassified bacteria (dead or alive) and taxonomic characterization. Moreover, both 454 pyrosequencing and Illumina display sequencing error rates; pyrosequencing is associated with relatively more insertions and deletions, whereas Illumina has more mismatches [62, 63]. Therefore, it is crucial to consider standardized control sequences to estimate the exact error rate for each experiment. Additionally, despite the fact that sequencing of $16 \mathrm{~S}$ rRNA provides insight into overall microbiota composition, it does not provide data on their functions and interactions. Finally, it should be noted that it was recently found that in certain conditions, next-generation sequencing data were found to be less representative of the 'real' microbiota than culture-based methods [64].

\section{Metagenomics and metabolomics}

As opposed to sequencing of marker genes such as the $16 \mathrm{~S}$ rRNA gene, sequencing of the entire genomic content of microorganisms (the 'microbiome') provides more specific information on their potential functional roles. These metagenomic or metatranscriptomic analyses, referred to as shotgun sequencing, are especially appropriate for linking microbial communities with functional potential and activity in the human gut. In addition, metabolomics- the quantitative assessment of metabolic responses of organisms to genetic or pathophysiological changes-is a powerful approach and also key to unraveling specific hostmicrobe interactions. Metagenomic approaches relate the microbiome to phenotype changes in disease, whereas metabolomic approaches relate the metabolic profile with disease phenotypes [65]. The latter analyses are principally based on proton nuclear magnetic resonance (1H-NMR) spectroscopy and mass spectrometry methods. Ideally, these analytical techniques should be complemented for the most detailed characterization of microbial metabolites obtained from feces, blood, urine, or intestinal tissues. In contrast to just sequencing the microbiota, shotgun-sequencing and metabolomics require direct freezing of fecal samples.

\section{Analysis with bioinformatics}

16S rRNA gene sequences can be analyzed with several tools, especially with QIIME [66] and Mothur software [67], which are reviewed in detail elsewhere [65, 68, 69]. These approaches produce phylogenetic trees and assessments of bacterial diversity within samples ( $\alpha$-diversity) and between samples ( $\beta$-diversity). To this end, sequences are clustered into taxonomic groups, referred to as operational taxonomic units (OTUs), by comparing them across samples (de novo) or to reference data such as Greengenes [70]. Importantly, a reference-based approach provides a more straightforward interpretation and enables the comparison of data with different sequenced regions of the $16 \mathrm{~S}$ rRNA gene. However, a crucial consideration and limitation of this technique is the common existence of unclassified bacteria in these databases and the complex taxonomic clustering including discrimination of related bacterial types. To reduce the effect of sequencing errors, it is recommended to disable the formation of new clusters with sequences that were not detected in any reference database. Fortunately, the increasing developments in the field of microbiota contribute to a high rate of classifying and discovering novel bacteria species. 


\section{Conclusion and perspectives}

Our progressive knowledge of the role of the gut microbiota in NAFLD is rapidly expanding due to improved DNA sequencing techniques. However, currently available studies show a marked discrepancy in results which is likely related to small sample size, variability in patient cohorts, and limited phenotyping of liver disease. Different sampling techniques and analysis methods likely also underlie the lack of consistent data. The key challenge now is to execute large-scale prospective studies with carefully phenotyped subjects; sequential samples should be obtained to demonstrate causality of gut microbiota changes in the etiology of NAFLD/NASH. In addition, diet, medicine use, and sampling methods should be well documented and considered before drawing conclusions. Importantly, metatranscriptomic and metabolic approaches are lacking and are urgently needed to assess the specific functional role of a certain microbial community. Providing insight into these aspects will help in understanding NAFLD pathophysiology and might eventually yield noninvasive biomarkers. However, next-generation sequencing techniques are currently not able to characterize the entire microbiota and their reproducibility has to be increased to use the microbiome as a diagnostic biomarker. Novel molecular methods, such as the promising IS-pro technique, may contribute to this [71]. Despite these challenges and the fact that the progression of NAFLD relies on multiple hits, the intestinal microbiota appears to represent an important factor that contributes to several aspects of NAFLD, and should be considered in any future mechanistic study.

Compliance with ethical requirements and Conflict of interest This article does not contain any studies with human or animal subjects. Niels van Best, Peter L. Jansen, and Sander S. Rensen declare that they have no conflict of interest.

Open Access This article is distributed under the terms of the Creative Commons Attribution 4.0 International License (http:// creativecommons.org/licenses/by/4.0/), which permits unrestricted use, distribution, and reproduction in any medium, provided you give appropriate credit to the original author(s) and the source, provide a link to the Creative Commons license, and indicate if changes were made.

\section{References}

1. Marchesini G, Brizi M, Bianchi G, Tomassetti S, Bugianesi E, Lenzi M, et al. Nonalcoholic fatty liver disease: a feature of the metabolic syndrome. Diabetes 2001;50:1844-1850

2. Cohen JC, Horton JD, Hobbs HH. Human fatty liver disease: old questions and new insights. Science 2011;332:1519-1523

3. Caldwell S, Argo C. The natural history of non-alcoholic fatty liver disease. Dig Dis 2010;28:162-168
4. Finelli C, Tarantino G. Nonalcoholic fatty liver disease, diet and gut microbiota. EXCLI J 2014;13:461-490

5. Day CP. Genetic and environmental susceptibility to non-alcoholic fatty liver disease. Dig Dis 2010;28:255-260

6. Brun P, Castagliuolo I, Di Leo V, Buda A, Pinzani M, Palu G, et al. Increased intestinal permeability in obese mice: new evidence in the pathogenesis of nonalcoholic steatohepatitis. Am J Physiol Gastrointest Liver Physiol 2007;292:G518-G525

7. Wigg AJ, Roberts-Thomson IC, Dymock RB, McCarthy PJ, Grose RH, Cummins AG. The role of small intestinal bacterial overgrowth, intestinal permeability, endotoxaemia, and tumour necrosis factor alpha in the pathogenesis of non-alcoholic steatohepatitis. Gut 2001;48:206-211

8. Zhu L, Baker SS, Gill C, Liu W, Alkhouri R, Baker RD, et al. Characterization of gut microbiomes in nonalcoholic steatohepatitis (NASH) patients: a connection between endogenous alcohol and NASH. Hepatology 2013;57:601-609

9. Mouzaki M, Comelli EM, Arendt BM, Bonengel J, Fung SK, Fischer SE, et al. Intestinal microbiota in patients with nonalcoholic fatty liver disease. Hepatology 2013;58:120-127

10. Raman M, Ahmed I, Gillevet PM, Probert CS, Ratcliffe NM, Smith S, et al. Fecal microbiome and volatile organic compound metabolome in obese humans with nonalcoholic fatty liver disease. Clin Gastroenterol Hepatol 2013;11:868.e1-3-875.e1-3

11. Weinstock GM. Genomic approaches to studying the human microbiota. Nature 2012;489:250-256

12. Nieuwdorp M, Gilijamse PW, Pai N, Kaplan LM. Role of the microbiome in energy regulation and metabolism. Gastroenterology 2014;146:1525-1533

13. Tremaroli V, Backhed F. Functional interactions between the gut microbiota and host metabolism. Nature 2012;489:242-249

14. Vos B, Moreno C, Nagy N, Fery F, Cnop M, Vereerstraeten P, et al. Lean non-alcoholic fatty liver disease (Lean-NAFLD): a major cause of cryptogenic liver disease. Acta Gastroenterol Belg 2011;74:389-394

15. Everard A, Belzer C, Geurts L, Ouwerkerk JP, Druart C, Bindels LB, et al. Cross-talk between Akkermansia muciniphila and intestinal epithelium controls diet-induced obesity. Proc Natl Acad Sci USA 2013;110:9066-9071

16. De Minicis S, Rychlicki C, Agostinelli L, Saccomanno S, Candelaresi C, Trozzi L, et al. Dysbiosis contributes to fibrogenesis in the course of chronic liver injury in mice. Hepatology 2014;59:1738-1749

17. Romano KA, Vivas EI, Amador-Noguez D, Rey FE. Intestinal microbiota composition modulates choline bioavailability from diet and accumulation of the proatherogenic metabolite trimethylamine-N-oxide. mBio 2015;6:e02481

18. Mehedint MG, Zeisel SH. Choline's role in maintaining liver function: new evidence for epigenetic mechanisms. Curr Opin Clin Nutr Metab Care 2013;16:339-345

19. Bajaj JS, Heuman DM, Hylemon PB, Sanyal AJ, White MB, Monteith $\mathrm{P}$, et al. Altered profile of human gut microbiome is associated with cirrhosis and its complications. J Hepatol 2014;60:940-947

20. Bajaj JS, Hylemon PB, Ridlon JM, Heuman DM, Daita K, White $\mathrm{MB}$, et al. Colonic mucosal microbiome differs from stool microbiome in cirrhosis and hepatic encephalopathy and is linked to cognition and inflammation. Am J Physiol Gastrointest Liver Physiol 2012;303:G675-G685

21. Kakiyama G, Pandak WM, Gillevet PM, Hylemon PB, Heuman DM, Daita K, et al. Modulation of the fecal bile acid profile by gut microbiota in cirrhosis. J Hepatol 2013;58:949-955

22. Liu J, Wu D, Ahmed A, Li X, Ma Y, Tang L, et al. Comparison of the gut microbe profiles and numbers between patients with liver cirrhosis and healthy individuals. Curr Microbiol 2012;65:7-13 
23. Goris H, de Boer F, van der Waaij D. Kinetics of endotoxin release by gram-negative bacteria in the intestinal tract of mice during oral administration of bacitracin and during in vitro growth. Scand J Infect Dis 1988;20:213-219

24. Qin N, Yang F, Li A, Prifti E, Chen Y, Shao L, et al. Alterations of the human gut microbiome in liver cirrhosis. Nature 2014;513:59-64

25. Miele L, Valenza V, La Torre G, Montalto M, Cammarota G, Ricci R, et al. Increased intestinal permeability and tight junction alterations in nonalcoholic fatty liver disease. Hepatology 2009;49:1877-1887

26. Cotillard A, Kennedy SP, Kong LC, Prifti E, Pons N, Le Chatelier E, et al. Dietary intervention impact on gut microbial gene richness. Nature 2013;500:585-588

27. Le Chatelier E, Nielsen T, Qin J, Prifti E, Hildebrand F, Falony $\mathrm{G}$, et al. Richness of human gut microbiome correlates with metabolic markers. Nature 2013;500:541-546

28. Schnabl B, Brenner DA. Interactions between the intestinal microbiome and liver diseases. Gastroenterology 2014;146:1513-1524

29. Neuschwander-Tetri BA, Loomba R, Sanyal AJ, Lavine JE, Van Natta ML, Abdelmalek MF, et al. Farnesoid X nuclear receptor ligand obeticholic acid for non-cirrhotic, non-alcoholic steatohepatitis (FLINT): a multicentre, randomised, placebo-controlled trial. Lancet 2015;385:956-965

30. Sayin SI, Wahlstrom A, Felin J, Jantti S, Marschall HU, Bamberg $\mathrm{K}$, et al. Gut microbiota regulates bile acid metabolism by reducing the levels of tauro-beta-muricholic acid, a naturally occurring FXR antagonist. Cell Metab 2013;17:225-235

31. Holt JA, Luo G, Billin AN, Bisi J, McNeill YY, Kozarsky KF, et al. Definition of a novel growth factor-dependent signal cascade for the suppression of bile acid biosynthesis. Genes Dev 2003; 17:1581-1591

32. Inagaki $\mathrm{T}$, Choi $\mathrm{M}$, Moschetta $\mathrm{A}$, Peng $\mathrm{L}$, Cummins $\mathrm{CL}$, McDonald JG, et al. Fibroblast growth factor 15 functions as an enterohepatic signal to regulate bile acid homeostasis. Cell Metab $2005 ; 2: 217-225$

33. Li F, Jiang C, Krausz KW, Li Y, Albert I, Hao H, et al. Microbiome remodelling leads to inhibition of intestinal farnesoid $\mathrm{X}$ receptor signalling and decreased obesity. Nat Commun 2013;4:2384

34. Miyata M, Yamakawa H, Hamatsu M, Kuribayashi H, Takamatsu Y, Yamazoe Y. Enterobacteria modulate intestinal bile acid transport and homeostasis through apical sodium-dependent bile acid transporter (SLC10A2) expression. J Pharmacol Exp Ther 2011;336:188-196

35. Miyata M, Takamatsu Y, Kuribayashi H, Yamazoe Y. Administration of ampicillin elevates hepatic primary bile acid synthesis through suppression of ileal fibroblast growth factor 15 expression. J Pharmacol Exp Ther 2009;331:1079-1085

36. Begley M, Gahan CG, Hill C. The interaction between bacteria and bile. FEMS Microbiol Rev 2005;29:625-651

37. Islam KB, Fukiya S, Hagio M, Fujii N, Ishizuka S, Ooka T, et al. Bile acid is a host factor that regulates the composition of the cecal microbiota in rats. Gastroenterology 2011;141:1773-1781

38. Inagaki T, Moschetta A, Lee YK, Peng L, Zhao G, Downes M, et al. Regulation of antibacterial defense in the small intestine by the nuclear bile acid receptor. Proc Natl Acad Sci USA 2006;103:3920-3995

39. Fouts DE, Torralba M, Nelson KE, Brenner DA, Schnabl B. Bacterial translocation and changes in the intestinal microbiome in mouse models of liver disease. J Hepatol 2012;56:1283-1292

40. Yoshimoto S, Loo TM, Atarashi K, Kanda H, Sato S, Oyadomari $\mathrm{S}$, et al. Obesity-induced gut microbial metabolite promotes liver cancer through senescence secretome. Nature 2013;499:97-101
41. Khandekar MJ, Cohen P, Spiegelman BM. Molecular mechanisms of cancer development in obesity. Nat Rev Cancer 2011;11:886-895

42. Schaap FG, Trauner M, Jansen PL. Bile acid receptors as targets for drug development. Nat Rev Gastroenterol Hepatol 2014;11:55-67

43. Thomas C, Gioiello A, Noriega L, Strehle A, Oury J, Rizzo G, et al. TGR5-mediated bile acid sensing controls glucose homeostasis. Cell Metab 2009;10:167-177

44. Bernsmeier C, Meyer-Gerspach AC, Blaser LS, Jeker L, Steinert RE, Heim MH, et al. Glucose-induced glucagon-like peptide 1 secretion is deficient in patients with non-alcoholic fatty liver disease. PLoS One 2014;9:e87488

45. Chimerel C, Emery E, Summers DK, Keyser U, Gribble FM, Reimann F. Bacterial metabolite indole modulates incretin secretion from intestinal enteroendocrine L cells. Cell Rep 2014;9:1202-1208

46. Yadav H, Lee JH, Lloyd J, Walter P, Rane SG. Beneficial metabolic effects of a probiotic via butyrate-induced GLP-1 hormone secretion. J Biol Chem 2013;288:25088-25097

47. Cani PD, Lecourt E, Dewulf EM, Sohet FM, Pachikian BD, Naslain D, et al. Gut microbiota fermentation of prebiotics increases satietogenic and incretin gut peptide production with consequences for appetite sensation and glucose response after a meal. Am J Clin Nutr 2009;90:1236-1243

48. Hwang I, Park YJ, Kim YR, Kim YN, Ka S, Lee HY, et al. Alteration of gut microbiota by vancomycin and bacitracin improves insulin resistance via glucagon-like peptide 1 in dietinduced obesity. FASEB J. 2015. doi:10.1096/fj.14-265983

49. Wu ZW, Lu HF, Wu J, Zuo J, Chen P, Sheng JF, et al. Assessment of the fecal lactobacilli population in patients with hepatitis $\mathrm{B}$ virus-related decompensated cirrhosis and hepatitis B cirrhosis treated with liver transplant. Microb Ecol 2012;63:929-937

50. Zoetendal EG, von Wright A, Vilpponen-Salmela T, Ben-Amor $\mathrm{K}$, Akkermans AD, de Vos WM. Mucosa-associated bacteria in the human gastrointestinal tract are uniformly distributed along the colon and differ from the community recovered from feces. Appl Environ Microbiol 2002;68:3401-3407

51. Green GL, Brostoff J, Hudspith B, Michael M, Mylonaki M, Rayment N, et al. Molecular characterization of the bacteria adherent to human colorectal mucosa. J Appl Microbiol 2006; 100:460-469

52. de Carcer DA, Cuiv PO, Wang T, Kang S, Worthley D, Whitehall $\mathrm{V}$, et al. Numerical ecology validates a biogeographical distribution and gender-based effect on mucosa-associated bacteria along the human colon. ISME J 2011;5:801-809

53. Zhang Z, Geng J, Tang X, Fan H, Xu J, Wen X, et al. Spatial heterogeneity and co-occurrence patterns of human mucosal-associated intestinal microbiota. ISME J 2014;8:881-893

54. Musser JM, Gonzalez R. Efficacy of an anaerobic swab transport system to maintain aerobic and anaerobic microorganism viability after storage at $-80{ }^{\circ} \mathrm{C}$. J Vet Diagn Invest 2011;23:95-99

55. Wasfy M, Oyofo B, Elgindy A, Churilla A. Comparison of preservation media for storage of stool samples. J Clin Microbiol 1995;33:2176-2178

56. Tedjo D, Jonkers D, Savelkoul P, Masclee A, van Best N, Pierik $\mathrm{M}$, et al. The effect of sampling and storage on the fecal microbiota composition in healthy and diseased subjects. PLoS One 2015 (in press)

57. Woese CR. Bacterial evolution. Microbiol Rev 1987;51:221-271

58. Caporaso JG, Lauber CL, Walters WA, Berg-Lyons D, Lozupone CA, Turnbaugh PJ, et al. Global patterns of 16S rRNA diversity at a depth of millions of sequences per sample. Proc Natl Acad Sci USA. 2011;108(Suppl 1):4516-4522

59. Oc P, de Carcer DA, Jones M, Klaassens ES, Worthley DL, Whitehall VL, et al. The effects from DNA extraction methods on 
the evaluation of microbial diversity associated with human colonic tissue. Microb Ecol 2011;61:353-362

60. Yuan S, Cohen DB, Ravel J, Abdo Z, Forney LJ. Evaluation of methods for the extraction and purification of DNA from the human microbiome. PLoS One 2012;7:e33865

61. Turnbaugh PJ, Quince C, Faith JJ, McHardy AC, Yatsunenko T, Niazi F, et al. Organismal, genetic, and transcriptional variation in the deeply sequenced gut microbiomes of identical twins. Proc Natl Acad Sci USA 2010;107:7503-7508

62. Gilles A, Meglecz E, Pech N, Ferreira S, Malausa T, Martin JF. Accuracy and quality assessment of 454 GS-FLX Titanium pyrosequencing. BMC Genom 2011;12:245

63. Luo C, Tsementzi D, Kyrpides N, Read T, Konstantinidis KT. Direct comparisons of Illumina vs. Roche 454 sequencing technologies on the same microbial community DNA sample. PLoS One 2012;7:e30087

64. Dubourg G, Lagier JC, Armougom F, Robert C, Hamad I, Brouqui $\mathrm{P}$, et al. The gut microbiota of a patient with resistant tuberculosis is more comprehensively studied by culturomics than by metagenomics. Eur J Clin Microbiol Infect Dis 2013;32:637-645

65. Morgan XC, Huttenhower C. Meta'omic analytic techniques for studying the intestinal microbiome. Gastroenterology 2014;146: 1437-1448.e1
66. Caporaso JG, Kuczynski J, Stombaugh J, Bittinger K, Bushman FD, Costello EK, et al. QIIME allows analysis of high-throughput community sequencing data. Nat Methods 2010;7:335-336

67. Schloss PD, Westcott SL, Ryabin T, Hall JR, Hartmann M, Hollister EB, et al. Introducing mothur: open-source, platformindependent, community-supported software for describing and comparing microbial communities. Appl Environ Microbiol 2009;75:7537-7541

68. Fraher MH, O'Toole PW, Quigley EM. Techniques used to characterize the gut microbiota: a guide for the clinician. Nat Rev Gastroenterol Hepatol 2012;9:312-322

69. Tyler AD, Smith MI, Silverberg MS. Analyzing the human microbiome: a "how to" guide for physicians. Am J Gastroenterol 2014;109:983-993

70. McDonald D, Price MN, Goodrich J, Nawrocki EP, DeSantis TZ, Probst A, et al. An improved Greengenes taxonomy with explicit ranks for ecological and evolutionary analyses of bacteria and archaea. ISME J 2012;6:610-618

71. Budding AE, Grasman ME, Lin F, Bogaards JA, SoeltanKaersenhout DJ, Vandenbroucke-Grauls CM, et al. IS-pro: highthroughput molecular fingerprinting of the intestinal microbiota. FASEB J 2010;24:4556-4564 